\title{
The Java System Dependence Graph
}

\author{
Neil Walkinshaw \\ 24th April 2003 \\ Technical Report: EFoCS-46-2003 \\ Department of Computer and Information Science \\ University of Strathclyde \\ Livingstone Tower \\ Glasgow, G1 1XH
}

\begin{abstract}
The Program Dependence Graph was introduced by Ottenstein and Ottenstein in 1984 [14]. It was suggested to be a suitable internal program representation for monolithic programs, for the purpose of carrying out certain software engineering operations such as slicing and the computation of program metrics. Since then, Horwitz et $a l$. have introduced the multi-procedural equivalent System Dependence Graph [9]. Many authors have proposed object-oriented dependence graph construction approaches $[11,10,20,12]$. Every approach provides its own benefits, some of which are language specific. This paper is based on Java and combines the most important benefits from a range of approaches. The result is a Java System Dependence Graph, which summarises the key benefits offered by different approaches and adapts them (if necessary) to the Java language.
\end{abstract}

\section{Introduction}

Analysing and representing software in terms of its internal dependencies is important for a variety of software engineering applications. These include operations such as slicing and the computation of program metrics. The program dependence graph represents these dependencies, where vertices are program elements and edges represent dependencies between them [14]. There have been several approaches to building graphs for different programming paradigms and languages. The Java System Dependence Graph (JSDG) summarises aspects of object-oriented programming that previous work has focused on and presents a practical approach to its construction.

Ottenstein and Ottenstein first suggested that dependence graphs could be used for software engineering operations in 1984 [14]. They proposed a graph which was capable of representing a program consisting of a single block of sequentially executed code. To enable the application of these operations to multi-procedural programs, Horwitz et al. introduced the System Dependence Graph, which represents every procedure as an individual dependence graph. The procedure dependence graphs are linked to a central dependence graph, which represents the main program [9].

There have been several proposed modifications to the system dependence graph, attempting to enable the representation of object-oriented programs. Such approaches must be able to cope with properties such as polymorphism, dynamic binding and inheritance. Larsen and Harrold proposed a graph capable of representing these features for $\mathrm{C}++$ programs [11]. This was modified by Kovács et al. and Zhao, to enable the representation of Javaspecific features such as interfaces, packages and single inheritance $[10,20]$. Liang and Harrold also augmented Larsen and Harrold's graph to distinguish data members in parameter objects, eliminating superfluous dependencies at callsites and hence increasing the accuracy of graph-based operations [12].

This paper presents a Java-based graph that encapsulates the benefits offered by the approaches mentioned above. It presents the graph construc- 
tion from a practical perspective and provides an example which demonstrates that the approach presented is viable. Although dependence analysis is an established area, the JSDG enables static analysis to be carried out on a graph which will produce more accurate results than other static Java dependence graphs, because it can represent abstract classes which need not necessarily be interfaces and it can distinguish data members in parameter objects.

The next section introduces the JSDG by presenting its individual components. Examples of various concepts which are included in the graph are taken from a single larger program which is given in the appendix. This is useful, because it puts the various individual illustrations into context. Section three analyses the graph from a more practical perspective. It identifies the steps needed for the construction of the graph. Section four analyses potential research areas that could benefit from the graph and introduces some practical problems that could arise if the represented program contains features such as threads and exceptions. Section five provides a conclusion and summary.

\section{The JSDG}

The abbreviation 'JSDG' is the same as the abbreviation used by Zhao [20]. The difference is, that Zhao's 'JSDG' stands for 'Java Software Dependence Graph', and the 'JSDG' in this paper stands for 'Java System Dependence Graph'. This can be seen as an extension to Zhao's JSDG, where a different mechanism is implemented for dealing with polymorphism and the representation of abstract classes is extended beyond interfaces.

A JSDG is a multigraph which maps out control and data dependencies ${ }^{1}$ between the statements of a Java program. Statements are categorised according to whether they contribute to the structure of a program (i.e. they are headers representing methods, classes, interfaces and packages) or the program's behaviour (i.e. they belong to a method body). Each category is represented differently on the graph. When these different graphs are combined, they provide a graph-based program representation, which is suitable as a basis for a range of software engineering applications.

The dependence graph is a complex construct and is intended as an internal program representation, not a visual one. It is difficult to visualise a graph which is composed of such a large number of different types of nodes and edges. This can however be partially facilitated by interpreting the JSDG as a layered architecture, where certain vertices on one layer are visible only to adjacent layers [16].

Depending on the application the dependence graph is intended for, not all of the nodes and edge types are required. The complexity of the graph can be reduced depending on the context in which it is applied. For example, if we intend to slice the dependence graph, any nodes or edges concerned with Java interfaces can be omitted.

\subsection{A Language-specific Representa- tion}

Object-oriented representations proposed by Larsen and Harrold and Liang and Harrold [11, 12] generate the dependence graph from $\mathrm{C}++$. Several of the differences between $\mathrm{C}++$ and Java require different edges or nodes in the graph. Its construction relies on the fact that it is possible to perform some preliminary control, data and call flow analysis on a given Java program, in order to build a skeletal version of the graph. Given that this framework is established, other nodes relating to the program structure (e.g. method and class vertices) are added. The accuracy of any traversal algorithm which operates on the JSDG (e.g. a slicing algorithm) depends on the accuracy of the flow analysis performed in the preprocessing stage.

\subsection{Statements}

A statement represents the lowest layer in the JSDG. It is an atomic construct representing a single expression in the source code of the program. A statement representing a call to another method (a callsite) requires a special representation and is described in section 2.4.1. Livadas and Croll suggest that accuracy of a slice on a dependence graph could be improved by increasing the granularity of the SDG to parse-tree level [13].

Java provides a more intuitive way to subdivide statements; when a Java program is compiled, it is translated into an intermediate, platformindependant format called the bytecode. There are several bytecode manipulation and analysis tools

\footnotetext{
${ }^{1}$ A control dependence $A \rightarrow_{c} B$ exists, if the execution of a statement $B$ relies on the execution of a predicate statement $A$. A data dependence $A \rightarrow_{d} B$ exists, if the execution of a statement $B$ references a variable which is defined / modified in a statement $A$.
} 
available (e.g. Soot ${ }^{2}$ and BCEL $^{3}$ ), which would make data and control flow analysis between individual bytecode instructions possible. For the sake of illustration, we will only consider the source-code statement level construction of the graph.

\subsection{Method Dependence Graph}

The method dependence graph (MDG) represents a single method or procedure in a program. It is the next layer up from the statement layer. MDGs are represented similarly in most $\mathrm{OO}$ dependence graph approaches $[10,11,12,20]$. The method entry vertex is connected to any other vertices belonging to the method via control dependence edges.

Parameter passing is modelled by introducing actual and formal variables. On the calling side, actual-in and actual-out vertices are tagged to copy each variable to and from its temporary location as required. The called method contains formal-in and formal-out vertices, which copy parameter variables from and to these temporary locations respectively. Parameter-in edges connect actual-in and formal-in vertices, while parameter-out edges connect formalout and actual-out vertices.

Further formal vertices are connected to the method entry vertex to account for instance variables which may be referenced or modified during the execution of the method. All formal vertices are connected to the method entry vertex and all actual vertices are connected to the callsite via control dependence edges. The flow of data within a method, to its actual-in and formal-out vertices and from its actual-out and formal-in vertices, is indicated by data dependence edges. The call dependence edge indicates the link between the callsite and the method being called.

Figure 1 illustrates an example of a simple method which adds two integers. To put this example into context, see the call from node C23 to E26 in appendix C. The method is represented by a method entry vertex (private int add(int $\mathrm{c}$, int d)), which is connected to statement vertices (int result $=c+d$ and return result) and formalin and formal-out vertices $\left(\mathrm{C}=\mathrm{C} \_\right.$in, $\mathrm{d}=\mathrm{d}$ _in and result_out=result) via control dependence edges (plain arrows). The callsite (int added=add(a,b)) belongs to another method and is connected to its actual-in and actual-out vertices (c_in $=a, d \_$in $=b$ and added=result_out) via control dependence edges. The call dependence edge from the callsite

\footnotetext{
${ }^{2}$ http://www.sable.mcgill.ca/software/

${ }^{3}$ http://jakarta.apache.org/bcel/
}

to the method entry vertex is represented by a dotted arrow. The actual-in vertices are connected to the formal-in vertices via parameter-in edges (dashed lines). The formal-out vertex is connected to the actual-out vertex via a parameter-out edge (dashed line). Data dependencies within the method (e.g. from $\mathrm{C}=\mathrm{C}$ _ in to int result $=\mathrm{C}+\mathrm{d}$ ) are represented by data dependence edges (dashed lines). A full legend for all of the examples featured in this paper is provided in appendix A.

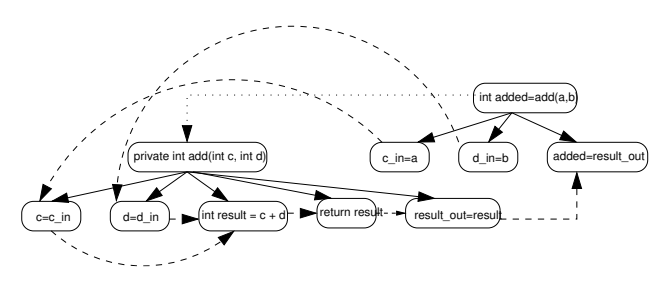

Figure 1: Example of a simple method call (extracted from appendix - call from node C26 to E29)

\subsection{Class Dependence Graph}

The class dependence graph (CIDG) represents the classes in a program [11]. It is the next layer up from the MDG layer. For every class, there exists a class entry vertex, which is connected to the method entry vertices of its methods via class membership edges. These membership edges can be tagged as either public, protected or package (default) to indicate their visibility [10]. If one class inherits from another, they are linked by a class dependence edge. The class entry vertex is connected to its data members via data member edges.

Figure 2 shows the CIDG of classes SimpleCalc and AdvancedCalc (see appendix B). Inheritance is indicated by the class dependence edge which passes between them. Note that although AdvancedCalc inherits all of the data members and methods belonging to SimpleCalc (apart from its constructors), it only needs to be linked to its own specific data members and methods. Inherited data members and methods can simply be computed by traversing up the class dependence edge and along the class membership / data member edges of SimpleCalc [10]. 


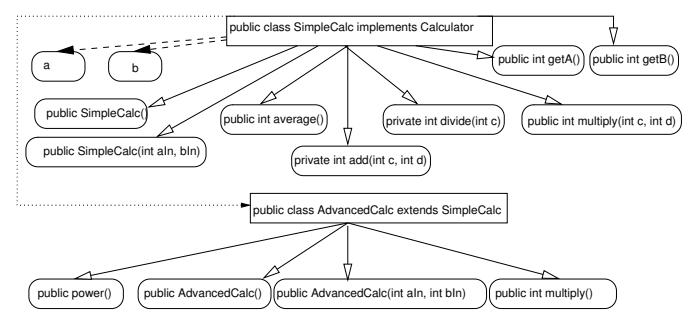

Figure 2: The CIDGs of the SimpleCalc and AdvancedCalc classes from appendix B (see nodes CE17 and CE46)

\subsubsection{Object Representation and Polymor- phism}

The JSDG represents different instances of a class individually; this enables dependence graph operations such as slicing to take individual objects into consideration [12]. A statement vertex $v$ which references an object is expanded into a tree depending on the context in which $v$ is used. The examples (figures 3-6) are taken from the calculator example given in appendices $\mathrm{B}$ and $\mathrm{C}$. The following four sections illustrate these possible expansions:

1. $v$ is a parameter vertex representing a statically typed ${ }^{4}$ object: $v$ is expanded into a tree. Figure 3 illustrates the callsite for getStats(e), given that it can only accept objects of the type AdvancedCalc.

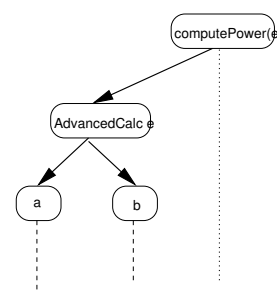

Figure 3: Example of single-typed parameter object (see node C9 in appendix)

2. $v$ is a parameter vertex representing a dynamically typed ${ }^{5}$ object: $v$ is connected to a child vertex for each possible object type and expands each child vertex into a tree containing data members belonging to that object. In figure 4 , e can either be of types SimpleCalc or AdvancedCalc.

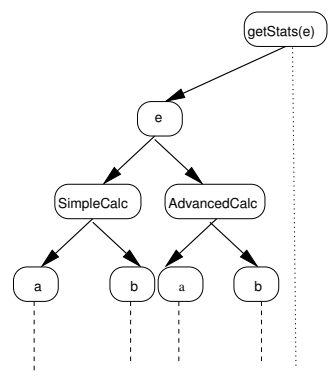

Figure 4: Example of polymorphic parameter object (see node C11 in appendix)

3. $v$ is a callsite vertex and the method being called is defined in a statically typed object: Because the implementation of the method can be determined statically, the callsite can simply be expanded by adding the actual-in and actual-out vertices. Note that, although the method does not have any parameters, we still need to represent the object data members as actual-in vertices, because they represent the instance variables referenced by the method. Figure 5 illustrates a call to power() contained in the statically typed AdvancedCalc object.

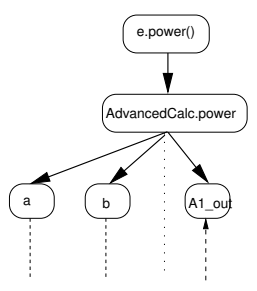

Figure 5: Example of a call to a method in a singletyped object (A1_out is the actual-out vertex ) (see node $\mathrm{C} 16 \mathrm{a}$ in appendix)

4. $v$ is a callsite vertex and the method being called is defined in a dynamically typed object: $v$ points to a vertex representing the object defining the method being called. This is further expanded into a tree where the branches represent the candidate types. These are further expanded to reveal the actual-in and actual-out vertices for the (potentially different) method implementations and linked to the method entry vertices via call edges. In

\footnotetext{
${ }^{4}$ The object type can be determined statically, without running the program

${ }^{5}$ The object type can only be determined dynamically
} 
figure 6, the multiply() implementation in AdvancedCalc is different to the one in SimpleCalc. The Java interpreter can only dynamically determine which implementation to execute.

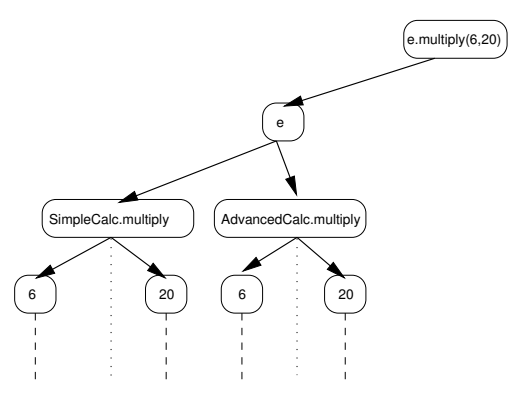

Figure 6: Example of a call to a method in a polymorphic object (see node S12a in appendix)

In every case, an object is expanded to reveal its data type(s). These are further expanded to represent their respective data members. If a data member happens to be another object, this must further be expanded to reveal its type(s) etc. This can become problematic if the object is defined recursively. To address this issue, Liang and Harrold employ $k$ limiting (the tree is only expanded to a level $k$ ) [12].

\subsection{Interface Dependence Graph}

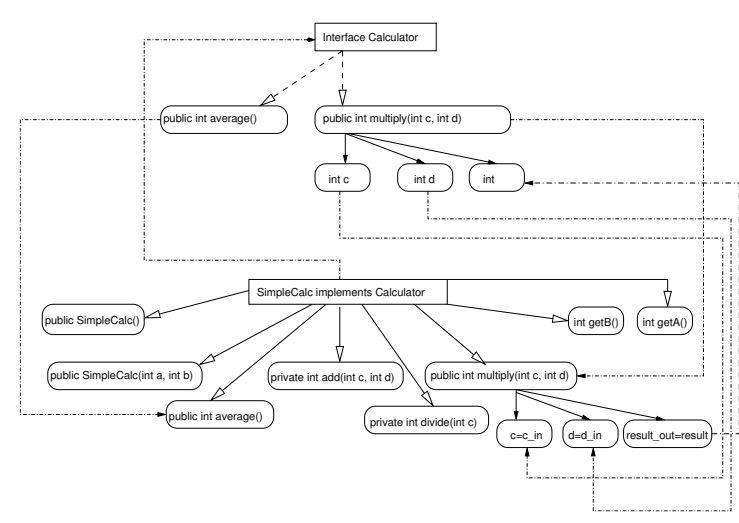

Figure 7: The InDG (see node IE43 in appendix)

The Java interface has been represented by both Kovács et al. and Zhao [10, 20]. Its role is to specify the signatures of the methods which must be implemented by any object implementing the interface.
Neither approach considers the representation of abstract classes which are not interfaces. The JSDG represents abstract classes as well as interfaces by treating the interface as a special kind of abstract class.

The JSDG deviates from previous interface representations by treating the interface as a special kind of abstract class. Because abstract classes can contain method implementations, the use of callsites to represent abstract methods as proposed by Zhao [20] becomes unsuitable. Abstract methods are represented in the JSDG with method entry vertices. Both Kovács et al. and Zhao omit parameter-out vertices from abstract method declarations $[10,20]$. To fully represent a method signature, if a method returns a value (i.e. is not void), the JSDG connects the method entry vertex to a parameter-out vertex.

The interface dependence graph $(\operatorname{InDG})$ consists of an interface entry vertex which is connected to a set of method entry vertices representing its abstract methods via abstract member edges. The method entry vertices are connected to parameter vertices, which represent their input parameters $^{6}$. Each method entry vertex is connected to the method entry vertex of the method implementing it by an implement abstract method edge. If a class implements an interface, the class is connected to the interface by an implements edge. If a class $C 1$ extends class $C 2$, and $C 2$ implements an interface, $C 1$ will automatically implement that interface as well. $C 1$ does not to be connected to the interface by an implemented edge, as this is implicit in the inheritance hierarchy. Figure 7 illustrates the Calculator InDG, which is connected to the SimpleCalc class. The multiply(int $c$, int $d$ ) vertex has been expanded to reveal its formal vertices in order to illustrate how parameters from the interface are connected to their implementation counterparts.

\begin{abstract}
Classes An abstract method contains only the method signature and leaves its implementation to a subclass. If a class contains an abstract method, it must itself be declared abstract. Abstract classes cannot be instantiated. In $\mathrm{C}++$ the equivalent effect is achieved by including a pure virtual method $^{7}$ in the class. Because interfaces are themselves abstract, abstract classes are represented in a similar fashion. The interface entry vertex is replaced with a class entry vertex. The class entry vertex is connected to abstract methods via an
\end{abstract}

\footnotetext{
${ }^{6}$ These vertices do not need to be tagged to assign an input value to a temporary location, because the interface is abstract

${ }^{7} \mathrm{~A}$ pure virtual method is a method that is declared as virtual and does not include a method body, but is initialised as ' 0 '.
} 
abstract member edge. Abstract methods are connected to their implementations via implement $a b$ stract method edges, as they would be in an interface. Non-abstract methods are represented as they would be in a normal CIDG. If a class entry vertex has at least one abstract member edge, it is an abstract class.

\begin{abstract}
Absence of Virtual Methods In $\mathrm{C}++$, the inheritance structure is slightly more complicated than in Java. Methods which can be overridden and dynamically bound at run-time must be explicitly marked as 'virtual'. In Java, it is simply presumed that any derived class which contains a method with the same signature as a method in a superclass overrides all definitions further up the inheritance hierarchy. Because Liang and Harrold base their dependence graph on $\mathrm{C}++$, they require a more complex inheritance structure [12]. Because Java allows only single inheritance and does not feature virtual methods, the JSDG can adopt a simpler inheritance structure, where derived classes can simply reuse base-class method definitions [10] (its simplicity is illustrated in figure 2).
\end{abstract}

\subsection{Package Dependence Graph}

A package defines a collection of classes which are conceptually similar or are dedicated to a similar purpose. It is represented by a package dependence graph $(P a D G)[10,20]$. Packages are important in terms of slicing, because they are needed to accurately compute variable visibility. A package entry vertex represents the package, which is connected to each class and interface entry vertex belonging to the package via a package member edge.

\section{Constructing the Graph}

Ultimately, a Java System Dependence Graph (JSDG) must satisfy the following properties: It must

- Represent methods, classes, and packages $[10,20]$

- Represent abstract methods / classes and interfaces

- Represent individual objects (it must be able to correctly represent polymorphic parameters calls to polymorphic objects) [12]
- Represent single inheritance (class hierarchy) [10]

The JSDG pools together the benefits of several previous dependence graph approaches. It provides a new representation for interfaces and abstract classes and combines the single-inheritance representation presented by Kovács et al. with the representation for methods, classes and packages proposed by Kovács et al. and Zhao [10, 20]. It also represents individual objects and can distinguish data members in parameter objects [12]. The pre-processing stage is beyond the scope of this document, but some important features are discussed briefly. The graph construction proceeds as follows:

\section{Construct MDGs}

(a) Pre process each method to ascertain callsites

(b) Expand objects

(c) Build data dependencies for data members

(d) Connect MDG nodes to a class node

\section{Construct CIDG}

3. Construct InDG

4. Construct PaDG

Pre-processing the Java program Building the JSDG requires prior control and data flow analysis. As discussed in section 2.1, this stage is instrumental in ensuring that the resulting JSDG and any operations on it are as accurate as possible. Chambers et al. propose an approach for accurately analysing data dependencies in Java programs which can handle exceptions, synchronization and memory consistency [3]. Tonella et al. propose a context and flow-insensitive Points-To Analysis (PTA) approach, which can reduce the size of the initial graph to increase the accuracy of operations such as slicing [18]. Grove et al. propose an approach to elicit call-graphs for OO programs [5].

A practical approach to carry out this prior analysis would be to use the Soot analysis framework, which provides several packages to analyse the Java byte-code. A problem with using Soot for this purpose is that it operates on the Java byte-code, not the source code. One line of source code usually constitutes several individual byte-code instructions ${ }^{8}$.

\footnotetext{
${ }^{8}$ Byte-code instructions are mapped to their respective source code line numbers in the LineNumberTable attribute of a class.
} 
This is made more difficult by the fact that if a class file is to be used in Soot, it has to be converted into one of several intermediate Soot formats (i.e. Baf, Jimple or Grimp), further confusing the mapping between intermediate instructions and source code line numbers. The upside of analysing a program at a byte-code level is that more precise results can be produced, especially in the case of slicing, where it is usually desirable to obtain a slice which is as accurate as possible.

\section{Construct MDGs}

1. (a) Processing Callsites In order to determine how the methods communicate with each other, each method must be processed individually. Methods to be processed are identified by traversing the call graph. Once a callsite has been identified it can be expanded (ref. 2.4.1). Once this is done, the call dependence edge is followed to determine the called method, where the appropriate formal-in and formal-out vertices are connected to its entry vertex. Conforming to Liang and Harrold's approach, we only add parameter vertices for parameters and global variables in the callee's GREF and $G M O D^{9}$.

A data dependence exists between vertices $A$ and $B$ if $A$ modifies / defines a variable which is referenced / used by $B$. To compute the data dependencies introduced by an object's data members, Liang and Harrold only associate the use of an object with a callsite if the called method is not a construction. An object definition is associated with a call vertex if the called method is not a destruction. In Java, destructors do not exist. In $\mathrm{C}++$, every object is destroyed when it goes out of scope or a pointer variable is deleted. In Java, unused objects are automatically destroyed in order to free up memory by way of a garbage collector. Java's closest equivalent of the destructor is the finalize () method $^{10}$. Hence, an object definition is associated with a call vertex if it is not a finalize $($ ) method.

1.(b) Expand Objects In order to expand objects, Liang and Harrold introduce the notion of an object-flow subgraph. This is a subgraph in the data dependence graph of a method, containing only the vertices that reference a given ob- ject. This subgraph is traversed, and each vertex $v$ is expanded as discussed in section 2.4.1. In the getStats(SimpleCalc e) method given in figure 8 , the vertices e, e.get $\mathrm{A}()$ and e.getB() belong to the object-flow subgraph and hence are expanded. [Note that it is necessary to expand the System.out.println... statement, because it is composed of two method calls, which must be represented separately.]

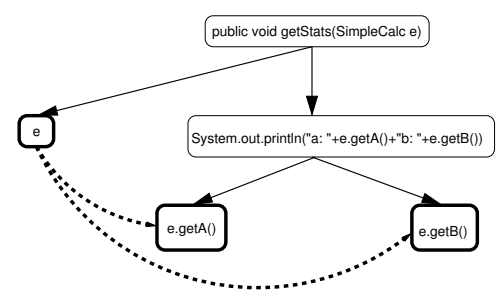

Figure 8: Example of an object-flow subgraph (vertices belonging to the graph are in bold)

1.(c) Build Data Dependencies for Data Members Once object vertices have been expanded, data dependencies must be established for the individual object data members. For a callsite $c$ in a subgraph, the definition set $D E F(c)$ of data members consists of c's actual-out vertices. The use set $U S E(c)$ is consists of $c$ 's actual-in vertices. If the call statement carries a parameter object, the object's data members must be added to the DEF and USE sets. For a parameter object, if the vertex defines the object ${ }^{11}$, the object's data members are added to the $D E F$ set. Similarly, if the vertex uses the object, the data members are added to the USE set. Having computed the DEF and USE sets, it is possible to generate the def-use chains as data dependencies.

2. Construct CIDG It is assumed that the class hierarchy is calculated as part of the pre-processing stage. For every class, a class entry vertex is generated, which is connected to the method entry vertices of methods belonging to that class via class membership edges. Kovács et al. use this connection to determine the visibility of the method within the class [10]. We adopt this approach as well, so that every class membership edge is tagged as either

\footnotetext{
${ }^{9} G M O D(m)$ is the set of non-local variables which can be modified within a method $m$ and $G R E F(m)$ is the set of non-local variables which can be referenced [1].

${ }^{10} \mathrm{In} \mathrm{C}++$, objects are explicitly destroyed as soon as they are out of scope. In Java, they are marked as unused when there are no longer any non-garbage references pointing to them. There is no way of guaranteeing when the garbage collector runs and when the finalize() is run. The timing of the garbage collection is also implementation-dependent, i.e. when the finalize() method is run depends in part on the Java implementation being used.

${ }^{11}$ An example of this would be i.compareTo(new Integer(5)); where i is of type Integer
} 
public, private, or protected. If a class A extends a class $\mathrm{B}, \mathrm{A}$ is connected to $\mathrm{B}$ via a class dependence edge. By connecting the classes in this manner, Java's single inheritance structure is emphasised. If a class contains an abstract method (i.e. the class is abstract), it is still represented by a conventional class entry vertex, but is connected to the abstract method via an abstract member edge. The abstract method is connected to its implementation in a subclass via an implement abstract method edge.

3. Construct InDG For every interface, there exists an interface entry vertex. This is connected to method entry vertices representing the abstract methods in the interface. These are each connected to their set of formal-in vertices. Each method is connected to its respective implementation's method entry vertex via an implements abstract method edge. The formal-in vertices connected to the interface method entry vertices are connected to their implementation counterparts via parameter-in edges.

4. Construct PaDG The PaDG is represented by a package entry vertex, which is connected to its class entry vertices and interface entry vertices via package edges. It is possible for a program to consist of package hierarchies. In this case, subpackages are connected to superpackages via package dependence edges. This is an important feature for multi-package programs, because it enables the accurate calculation of the visibility of classes.

\section{Operating on the JSDG}

Although this paper focuses on the graph itself, it makes sense to give the reader an idea of some of its potential benefits. The main application is slicing, which has been the focus of the majority of dependence graph based papers [9, 10, 11, 12, 14, 20]. In addition to slicing, Horwitz and Reps also propose that dependence graphs can be used to establish differences between two programs (program differencing) and to integrate changes carried out on one program into another similar program (program integration) [8]. The combination of data and control dependencies provides a useful basis for the calculation of program metrics [14]. It would also be interesting to investigate the usefulness of the JSDG with respect to software inspections. In object-oriented software inspections, delocalised software artifacts which are connected by control and data dependencies hamper code reviews, because the code inspec- tor is forced to jump from one part of the code to another [4].

\subsection{Slicing}

If the JSDG is to be sliced, it needs an additional edge called the summary edge. These represent the transitive flow of dependence across a callsite caused by both control and data dependencies. Such an edge connects an actual-in vertex to an actual-out vertex if the value associated with the actual-in vertex may affect the value associated with the actualout vertex. Figure 10 shows the same callsite example as figure 1, but adds transitive dependencies from $c \_i n=a$ to added=result_out and d_in $=b$ to added=result_out.

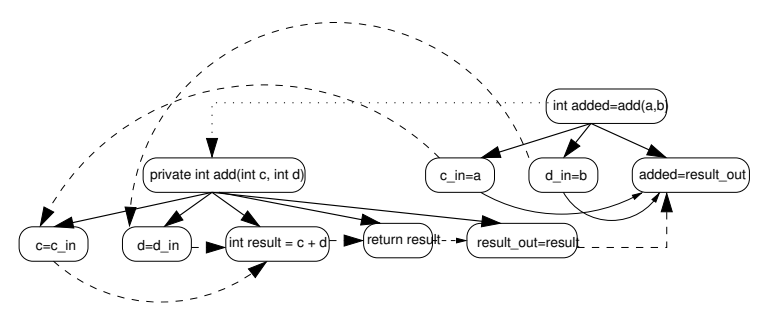

Figure 9: Example of method call with transitive edges between actual-in and actual-out vertices

The slicing algorithm proposed by Horwitz et al. is split into two phases. The first phase traverses backwards along control, call, parameter in and data dependence edges marking every graph vertex it passes. In the second pass, the algorithm traverses back from each marked vertex along control, parameter out and data dependence edges [9]. Liang and Harrold extended this algorithm to enable the slicing of individual objects [12]. An example of a slice according to the Horwitz et al. method is marked out in appendix C (shaded vertices belong to a slice taken from statement S25).

\subsection{Program Metrics}

Ottenstein and Ottenstein suggested that the dependence graph would be a suitable basis for the calculation of program metrics. The JSDG allows individual methods, classes or packages to be measured. This could be especially useful as a heuristic to software restructuring. If the complexity in a given area of the program exceeds a certain threshold, it could indicate that a refactoring (or other form of code restructuring) could be necessary. 
It would be interesting to expand on Weiser's original investigations into slicing based metrics [19]. Bieman and Ott propose the use of program slices to measure functional cohesion [2]. According to Riel, a good object-oriented designer strives for "tight cohesion within classes and loose coupling between classes" [15]. It would be useful to extend this measure to the object-oriented paradigm. The JSDG provides the basic representation for the computation of these slices.

\subsection{Software Inspections}

Dunsmore et al. state that delocalised software artifacts hamper object oriented code inspections [4]. Software artifacts become delocalised because object-oriented paradigm features such as inheritance, polymorphism and dynamic binding can cause code which is responsible for the execution of a single task to be dispersed throughout the program. These dispersed artifacts are all connected via some form of dependence (or chain of dependencies), which can be traced on the JSDG. Slicing could be used to statically determine possible paths of execution in the program, providing the inspector with a reading strategy for the inspection.

"Program understanding requires tracing chains of method invocations up and down the class hierarchy" [4]. The inspector must be able to abstract the high level function of a software module to verify that it conforms to its specification. Harman et al. propose a framework for combining slicing and concept assignment [6], which would significantly reduce this laborious aspect of inspections. Further research is required if this approach is to be made practical for object-oriented systems. The JSDG provides a useful basis for investigating the feasibility of extracting Executable Concept Slices (ECSs) for object-oriented programs.

\subsection{Practical Issues}

The graph has not been designed to incorporate exceptions and threads. Sinha et al. represent exceptions by adding vertices and edges around the try and catch clauses of an exception[17]. Hatcliff et al. study the slicing of multi-threaded programs, but do not specifically relate their solution to a program dependence graph representation [7].

Another problem that is prevalent amongst most static analysis techniques is that the graph produced is very substantial. This is due to the fact that a static approach must lay out every possible execution that could possibly be carried out by the program. The size can make the generation and storage of such a graph an expensive process. Depending on the purpose for which it is intended certain edges and vertices can be removed if they are not going to be used (i.e. interface related vertices and edges can be removed if the graph is only needed for slicing operations). If we are only interested in a given subset of methods in the program other parts could be sliced away.

\section{Conclusions}

This dependence graph provides a useful basis for the representation of Java programs. It enables several useful software engineering operations to be carried out as queries / manipulations on the graph, which offers greater speed and precision than conventional methods (Horwitz et al. illustrate the increase in precision when slicing the SDG as opposed to Weiser's conventional algorithm [9]). It provides a representation for interfaces and abstract classes and enables objects and object data members to be treated individually in any operation (e.g. the program can be sliced object by object). Now, it is possible to re-interpret the dependence graph applications as suggested by Ottenstein and Ottenstein and Horwitz et al. in terms of the OO paradigm. Several potential research areas concerning the JSDG have been proposed. The next logical step in making the JSDG a practical software engineering tool is to develop a tool which will automatically build an internal representation of a given Java program.

\section{References}

[1] J. Banning. An efficient way to find the side effects of procedure calls and the aliases of variables. In Conference Record of the Sixth Annual ACM Symposium on Principles of Programming Languages, pages 29-41, January 1979.

[2] J. Bieman and L. Ott. Measuring functional cohesion. IEEE Transactions on Software Engineering, 20(8):644-658, August 1994.

[3] C. Chambers, I. Pechtchanski, V. Sarkar, M. Serrano, and H. Srinivasan. Dependence analysis for java. In Workshop on Compilers for Parallel Computing, La Jolla, LA, August 1999.

[4] A. Dunsmore, M. Roper, and M. Wood. Object-oriented inspection in the face of delocalisation. In Proceedings of the 22nd International Conference on Software Engineering, Limerick, 2000.

[5] D. Grove, G. DeFouw, J. Dean, and C. Chambers. Call graph construction in object-oriented languages. In $O O P$ SLA '97 Conference Proceedings, 1997.

[6] M. Harman, N. Gold, R. Hierons, and D. Binkley. Code extraction algorithms which unify slicing and concept as- 
signment. In 9th IEEE Conference on Reverse Engineering (WCRE '02), Richmond, Virginia, USA, 2002.

[7] J. Hatcliff, J. Corbett, M. Dwyer, S. Sokolowski, and H. Zheng. A formal study of slicing for multi-threaded programs with jvm concurrency primitives. Technical Report 99-6, Kansas State University, March 1999.

[8] S. Horwitz and T. Reps. The use of program dependence graphs in software engineering. In Proceedings of the 14th International Conference on Software Engineering, 1992.

[9] S. Horwitz, T. Reps, and D. Binkley. Interprocedural slicing using dependence graphs. ACM Transactions on Programming Languages and Systems, 12(1):26-60, January 1990.

[10] G. Kovacs, F. Magyar, and T. Gyimothy. Static slicing of java programs. Technical Report TR-96-108, Research Group on Artificial Intelligence, Hungarian Academy of Sciences, Joesf Attila University, 1996.

[11] L. Larsen and M. Harrold. Slicing object oriented software. In 18th International Conference on Software Engineering, pages 495-505, March 1996.

[12] D. Liang and M. Harrold. Slicing objects using system dependence graphs. International Conference on Software Maintenance, pages 358-367, November 1998.

[13] P. Livadas and S. Croll. Program slicing. 1992.

[14] K. Ottenstein and L. Ottenstein. The program dependence graph in a software development environment. In Proceed- ings of the ACM SIGSOFT/SIGPLAN Software Engineering Symposium on Practical Software Development Environments, pages 177-184, 1984.

[15] A. Riel. Object-Oriented Design Heuristics. Addison Wesley, 1996.

[16] M. Shaw. Pattern Languages of Program Design 2, chapter Some Patterns for Software Architectures. Addison Wesley, 1996.

[17] S. Sinha, M. Harrold, and G. Rothermel. Systemdependence-graph-based slicing of programs with arbitrary interprocedural control flow. In Proceedings of the 21st International Conference on Software Engineering, May 1999.

[18] P. Tonella, G. Antoniol, R. Fuitem, and E. Merlo. Flow insensitive $\mathrm{C}++$ pointers and polymorphism analysis and its application to slicing. 19th International Conference on Software Engineering, pages 433-443, May 1997.

[19] M. Weiser. Program slicing. In Proc. 5th Int. Conference on Software Engineering, pages 439-449, New York, 1981. IEEE.

[20] J. Zhao. Applying program dependence analysis to java software. In Proc. Workshop on Software Engineering and Database Systems, pages 162-169, Taiwan, December 1998.

\section{A Legend}

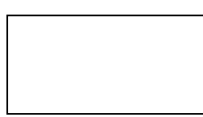

class / interface entry vertex

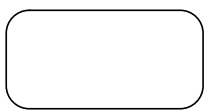

method entry vertex / statement vertex / formal or actual parameter in/out vertex

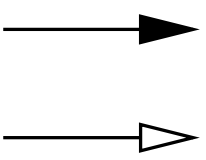

control dependence

belongs to (can be tagged to denote the visibility of a method) OR interface member edge

implements edge

data dependence edge

call / implements abstract method edge

Data member 


\section{B Example Code}

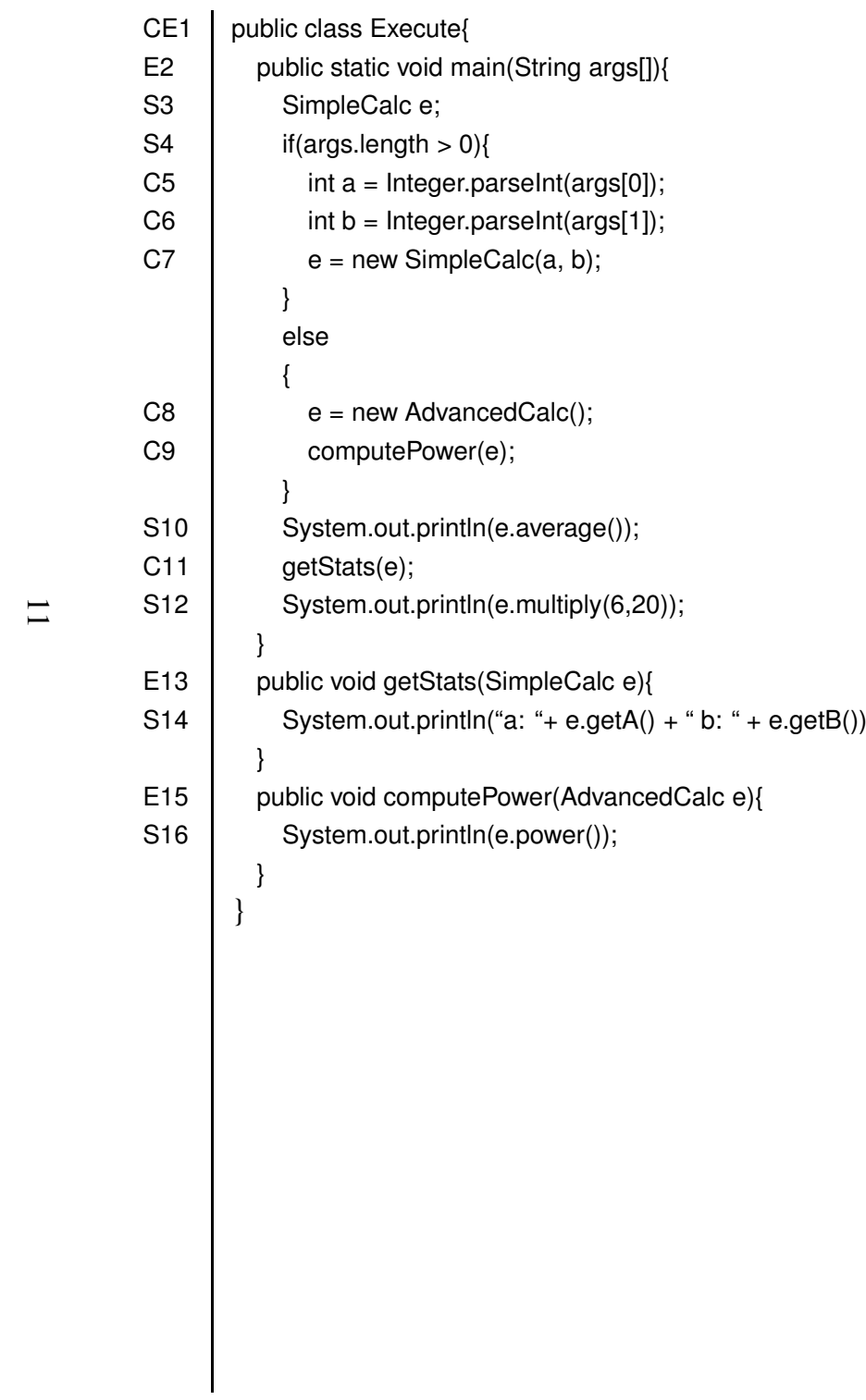

\begin{tabular}{|c|c|}
\hline CE17 & public class SimpleCalc implements Calculator\{ \\
\hline S18 & int a,b; \\
\hline E19 & public SimpleCalc()\{ \\
\hline S20 & $a=6$ \\
\hline S21 & $b=20$ \\
\hline E22 & public SimpleCalc(int aln, int bln)\{ \\
\hline S23 & $a=a l n$ \\
\hline $\mathrm{C} 24$ & b = multiply $(a, b l n)$ \\
\hline E25 & public int average ()\{ \\
\hline C26 & int added $=\operatorname{add}(a, b)$; \\
\hline $\mathrm{C} 27$ & int divided = divide(added); \\
\hline S28 & \}return divided; \\
\hline E29 & private int add(int $\mathrm{c}$, int $\mathrm{d})\{$ \\
\hline S30 & int result $=\mathrm{c}+\mathrm{d}$; \\
\hline S31 & return result; \\
\hline E32 & private int divide(int c) \{ \\
\hline S33 & int result = $\mathrm{c} / 2$ \\
\hline S34 & return result; \\
\hline E35 & protected int multiply(int c, int d) \{ \\
\hline S36 & for (int $\mathrm{i}=0 ; \mathrm{i}<\mathrm{c} ; \mathrm{i}++)\{$ \\
\hline S37 & \}$^{d=d+d ;}$ \\
\hline S38 & \}$^{\text {return d; }}$ \\
\hline E39 & public int get $A()\{$ \\
\hline S40 & \} return a; \\
\hline E41 & public int getB( $)\{$ \\
\hline S42 & \}$^{\text {return b; }}$ \\
\hline
\end{tabular}

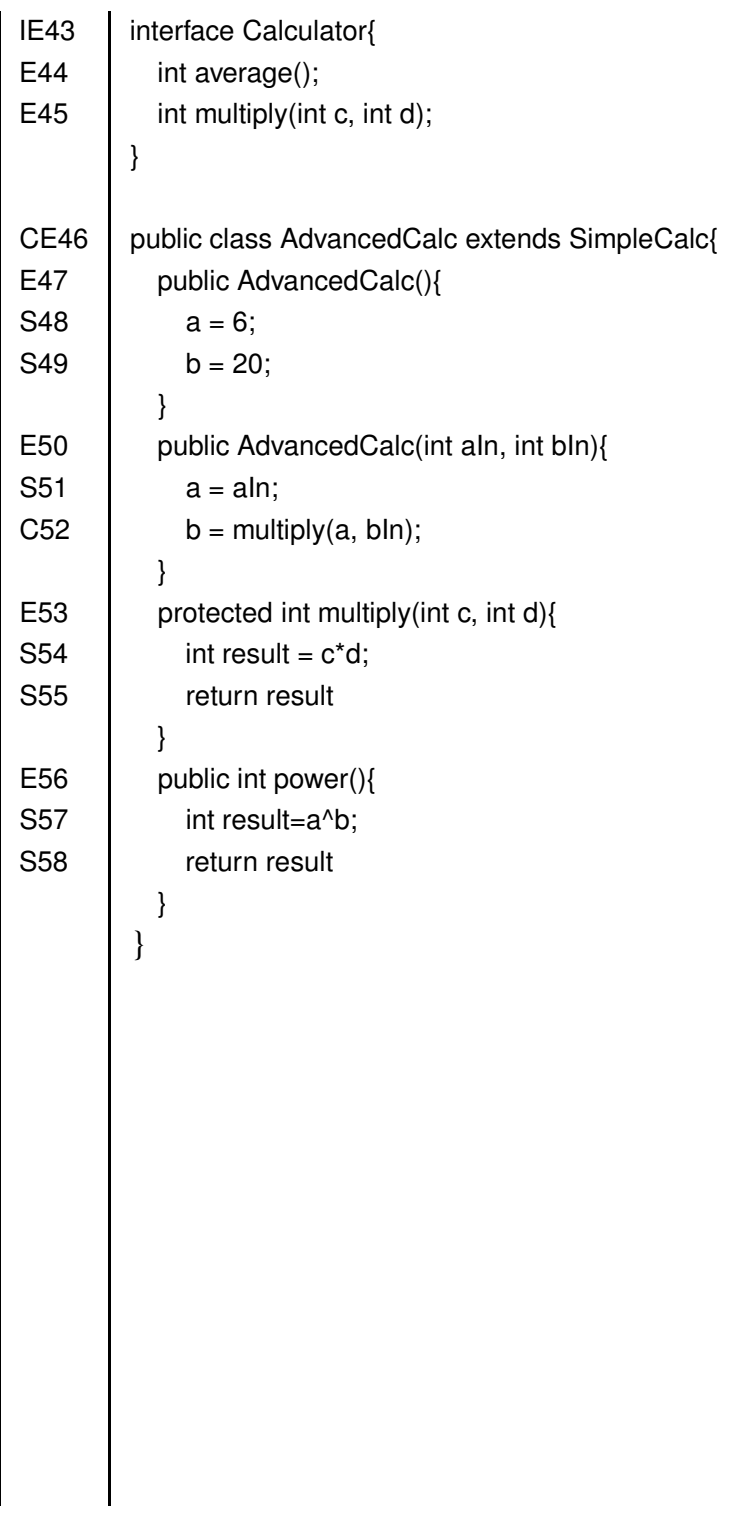




\section{Entire JSDG for Example Code}

A backwards-slice is demonstrated, taken from vertex S25 (return divided). This page is best viewed in colour. Vertices marked by the first phase of the slice are shown in blue (darker shade) and those marked by the second phase are shown in pink (lighter shade).

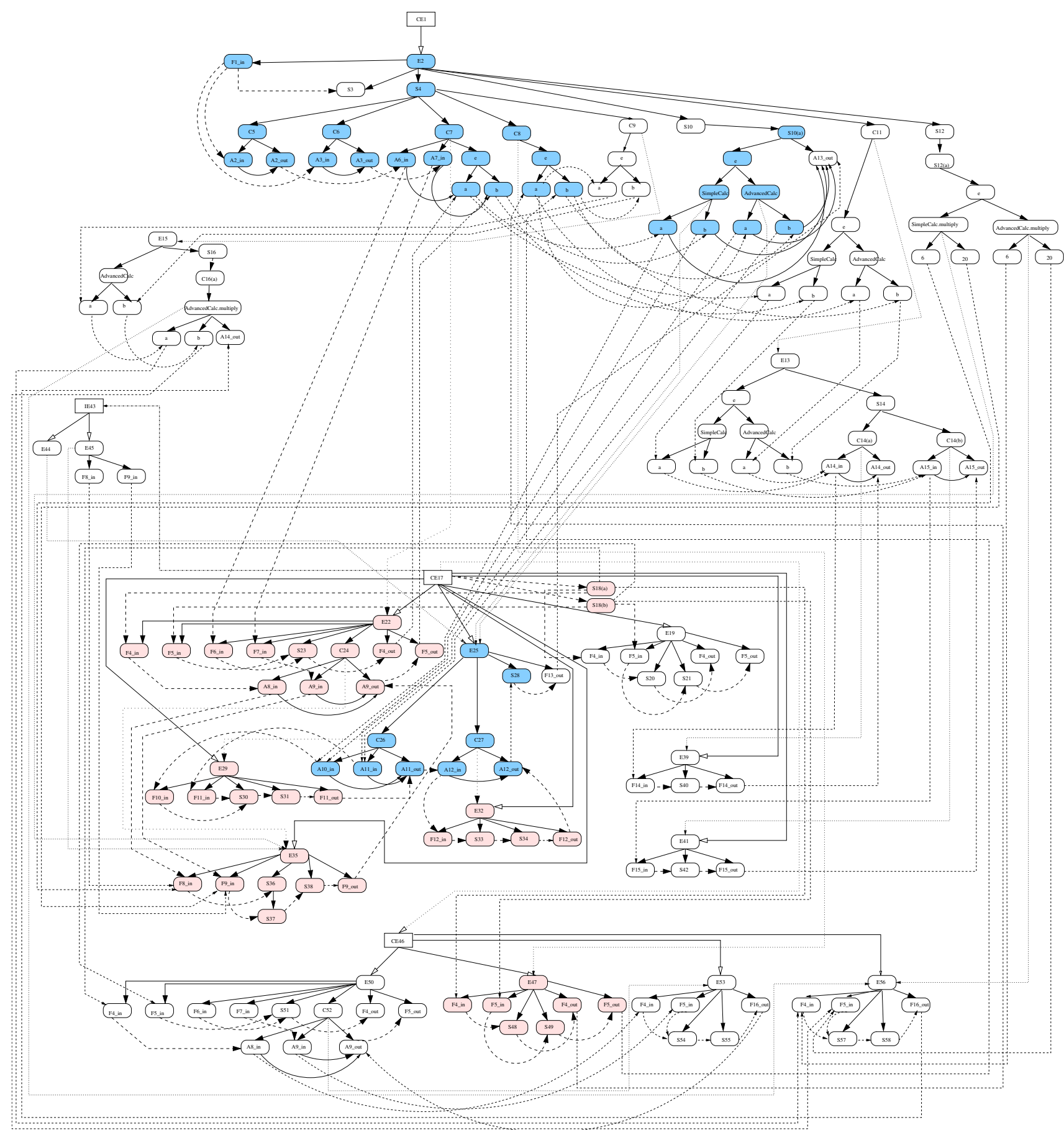

